\title{
TRATAMENTO DE EFLUENTE DE ABATEDOURO DE TILÁPIA COM ADIÇÃO DE MANIPUEIRA NA FASE ANÓXICA ${ }^{1}$
}

\section{ANA CAROLINA B. KUMMER ${ }^{2}$, LIDIANA DE ANDRADE ${ }^{3}$, SIMONE D. GOMES ${ }^{4}$, AJADIR FAZOLO ${ }^{5}$, SALAH D. M. HASAN ${ }^{6}$, FABIANO MACHADO ${ }^{7}$}

RESUMO: O objetivo deste trabalho foi avaliar a desnitrificação de efluente de abatedouro de tilápia, em reator em batelada. Para isto, foi avaliado o efluente gerado por uma indústria processadora de mandioca (manipueira) como fonte de carbono. Também foram avaliadas as condições de agitação do sistema. Os experimentos foram conduzidos em escala de laboratório, em que foram testados cinco níveis de agitação $(20 ; 32 ; 60 ; 88$ e $100 \mathrm{rpm})$ e cinco níveis de relação DQO/N (0,1; 1,0; 3,2; 5,4 e 6,3), configurando-se um planejamento do tipo Delineamento Composto Central Rotacional (DCCR), com quatro ensaios nos níveis +1 e -1 ; quatro ensaios nos níveis dos pontos axiais $(-1,414$ e $+1,414)$ e mais uma triplicata no ponto central $(0)$, totalizando 11 ensaios. Avaliaram-se o desempenho da desnitrificação através da remoção de nitrato (\%) e a remoção de nitrito $(\%)$. Durante o processo, também foram monitorados temperatura $\left({ }^{\circ} \mathrm{C}\right), \mathrm{pH}$ e alcalinidade $\left(\mathrm{mgCaCO}_{3} \cdot \mathrm{L}^{-1}\right)$. Os resultados mostraram que a relação DQO/N teve influência significativa, com intervalo de confiança de $95 \%$, sobre o processo de remoção de nitrato e nitrito, com faixa ótima de operação entre 3,2 e 5,4, cujas eficiências de remoção de nitrogênio foram de $100 \%$.

PALAVRAS-CHAVE: remoção biológica, desnitrificação, nitrato, nitrito, efluente de abatedouro de peixe, efluente de fecularia.

\section{TREATMENT OF TILAPIA SLAUGHTERHOUSE WASTEWATER WITH ADDITION OF CASSAVA IN ANOXIC STAGE}

\begin{abstract}
The objective of this work was to evaluate the denitrification of tilapia slaughterhouse wastewater, in a batch reactor. For this, it was evaluated the wastewater from a cassava processing industry (manipueira) as a carbon source. It was also evaluated the shaking conditions of the process. The experiments were carried out in laboratory series, where five shaking levels were tested $(20 ; 32 ; 60 ; 88$ e $100 \mathrm{rpm})$ as well as five COD/N ratio levels $(0.1 ; 1.0 ; 3.2 ; 5.4$ and 6.3), forming a planning as a Central Composite Rotational Design (CCRD), with four essays in levels +1 and -1 ; four essays on the axial points levels $(-1.414$ and +1.414$)$ and one more triplicate in the central point (0), which meant eleven essays. The denitrification performance through the nitrate removal $(\%)$ and nitrite removal $(\%)$ were evaluated. During this process, temperature $\left({ }^{\circ} \mathrm{C}\right), \mathrm{pH}$ and alkalinity $(\mathrm{mgCaCO} 3 . \mathrm{L}-1)$ were also observed. The results showed that the $\mathrm{COD} / \mathrm{N}$ ratio had significant influence, with a $95 \%$ of confidence interval, on the process of nitrate and nitrite removal, with a great operation range, from 3.2 to 5.4, whose nitrogen removal efficiencies were $100 \%$.
\end{abstract}

KEYWORDS: biologic removal, denitrification, nitrate, nitrite, fish wastewater from slaughterhouse, cassava wastewater.

\footnotetext{
${ }^{1}$ Extraído da dissertação de mestrado do primeiro autor.

${ }^{2}$ Eng ${ }^{\underline{a}}$ Agrícola, Mestre em Engenharia Agrícola, Doutoranda em Agronomia (Irrigação e Drenagem), Universidade Estadual Paulista "Júlio de Mesquita Filho", Faculdade de Ciências Agronômicas, Botucatu - SP, ackummer@hotmail.com.

${ }^{3}$ Bióloga, Mestre em Engenharia Agrícola, Universidade Estadual do Oeste do Paraná, UNIOESTE, Câmpus de Cascavel - PR, lidi_ana@hotmail.com.

${ }^{4}$ Prof. Adjunto, Centro de Ciências Exatas e Tecnológicas, Universidade Estadual do Oeste do Paraná, UNIOESTE, Câmpus de Cascavel - PR, simoned@unioeste.br.

${ }^{5}$ Prof. Adjunto, Universidade Tecnológica Federal do Paraná, UTFPR, Londrina - PR, afazolo@ @otmail.com.

${ }^{6}$ Prof. Dr., Universidade Estadual do Oeste do Paraná, UNIOESTE, Câmpus de Toledo - PR, salahdmh@ @mail.com.

${ }^{7}$ Eng $^{\mathrm{o}}$ Civil pela Universidade Estadual do Oeste do Paraná, UNIOESTE, Câmpus de Cascavel - PR, fmachado182@ hotmail.com

Recebido pelo Conselho Editorial em: 9-9-2008

Aprovado pelo Conselho Editorial em: 15-12-2010
} 


\section{INTRODUÇÃO}

O processamento industrial de tilápia no Brasil iniciou-se na década de 1990, no oeste do Paraná, priorizando-se apenas uma forma de beneficiamento, ou seja, filés de tilápia congelados, que permanece até os dias de hoje. Segundo KUBITZA (2000), a região Sul do Brasil contribui com $75 \%$ da produção nacional de tilápia, sendo o Estado do Paraná o maior produtor atualmente.

KIRSCHNIK (2007) destaca que o rendimento em filé de tilápia é baixo, cerca de 30 a 33\%, consequentemente é gerada grande quantidade de resíduos sólidos, além do significativo volume de efluente líquido, proveniente de processos de lavagem. Tendo em vista que esses efluentes apresentam matéria orgânica, gordura e elevada concentração de nitrogênio, apenas o emprego de tratamento primário e o secundário convencional não são suficientes para atingir os padrões de lançamento exigidos pelos órgãos ambientais. Assim, faz-se necessário o emprego de técnicas de tratamento de efluentes que minimizem os impactos gerados ao meio ambiente.

A descarga de efluentes, com tratamento insuficiente em corpos de água receptores, pode resultar em severos problemas ambientais, deterioração dos meios naturais e morte da fauna de rios e lagos.

Atualmente, tem sido crescente a preocupação com a remoção dos nutrientes das águas, fundamentada na necessidade de garantir a qualidade do efluente, bem como para proteger os corpos de água receptores.

O acúmulo de nutrientes em águas naturais pode causar eutrofização, com efeitos adversos, tais como proliferação de algas, diminuição da concentração de oxigênio dissolvido, formação de toxinas, problemas de odor, morte de peixes, efeitos nocivos à saúde humana, dificultando a autodepuração do corpo hídrico receptor.

Assim, as exigências ambientais quanto à qualidade do efluente da agroindústria preconizam a remoção de matéria orgânica e de nutrientes como prioridades dos tratamentos biológicos e físicoquímicos disponíveis e aplicados.

De acordo com FERREIRA (2000), os compostos nitrogenados podem ser removidos de efluentes por intermédio de tratamentos químicos, físicos ou biológicos. Os processos biológicos de remoção de nitrogênio apresentam-se eficazes e de fácil projeto e operação, quando comparados aos métodos físico-químicos. Assim sendo, os processos biológicos de remoção de nitrogênio estão encontrando crescentes aplicações e vêm substituindo os processos físico-químicos, com menores custos operacionais.

Para a remoção dos compostos de nitrogênio, a nitrificação é uma etapa intermediária, visto que, neste processo, acontece apenas a conversão do nitrogênio presente na água residuária, em compostos oxidados de nitrogênio. A etapa seguinte compreende o processo de desnitrificação, no qual os compostos oxidados de nitrogênio, nitrito e nitrato serão, então, transformados em nitrogênio gasoso que é devolvido à atmosfera (ISOLDI \& KOETZ, 2004).

Neste contexto, este trabalho teve como objetivo avaliar o processo de desnitrificação de efluente de abatedouro de tilápia, em reator em batelada, através da remoção biológica de nitrato e nitrito. Para isto, foi utilizado o efluente gerado por uma indústria processadora de mandioca (manipueira) como fonte de carbono, bem como foram testados diferentes níveis de agitação para o processo.

\section{MATERIAL E MÉTODOS}

O experimento foi conduzido no Laboratório de Saneamento Ambiental da Universidade Estadual do Oeste do Paraná - UNIOESTE - Câmpus de Cascavel.

O efluente a ser tratado foi proveniente da lagoa anaeróbia de uma indústria de abate de tilápia localizada na cidade de Toledo - PR. A indústria prioriza a obtenção de filés e diariamente abate cerca de 8 toneladas, o que gera, em média, 56.000 L de água residuária. Para cada ensaio 
experimental, $2 \mathrm{~L}$ de efluente eram submetidos à nitrificação, em um reator aeróbio operado em bateladas sequenciais, com volume útil de $2,5 \mathrm{~L}$, biomassa imobilizada e vazão de ar constante de $2 \mathrm{~L} \mathrm{~min}^{-1}$. A parede do reator foi revestida com espuma de poliuretano de $1 \mathrm{~cm}$ de espessura para dar suporte à biomassa nitrificante. $\mathrm{O}$ reator foi operado em ciclos de 24 horas, com monitoramento das concentrações de amônia, entre outros parâmetros, para verificar a conversão de nitrogênio às formas oxidadas (nitrito e nitrato).

A manipueira foi proveniente de uma indústria processadora de mandioca, localizada na cidade de Toledo - PR. O produto principal da industrialização é a fécula, o qual gera cerca de 100.000 L de água residuária por dia, tendo em vista que são utilizados $4 \mathrm{~L}$ de água por quilo de mandioca processada. A coleta da água residuária foi realizada na saída da indústria, antes do sistema de tratamento. As amostras foram preservadas à temperatura de $0^{\circ}$ a $4{ }^{\circ} \mathrm{C}$, para evitar a fermentação do líquido. Em laboratório, antes de ser caracterizada, a manipueira foi peneirada para eliminação de resíduos sólidos (presença de casca de mandioca) e submetida ao processo de sedimentação por 15 minutos, para eliminação dos sólidos suspensos (solo). Dessa forma, o resíduo líquido, utilizado como fonte de carbono no estudo, foi o sobrenadante resultante da sedimentação.

Para o processo de desnitrificação, foi utilizado um reator anóxico (não aerado) em batelada, construído com tubo de PVC, com $20 \mathrm{~cm}$ de altura e $15 \mathrm{~cm}$ de diâmetro, com volume útil de 2,5 L. $\mathrm{O}$ fundo do reator foi revestido com espuma de poliuretano de $1 \mathrm{~cm}$ de espessura para dar suporte e promover crescimento da biomassa responsável pela desnitrificação. O reator anóxico foi operado em ciclos de 4 horas e alimentado através de sifonação, com o efluente oriundo do processo de abate de peixes, previamente nitrificado em reator aeróbio. O reator foi adaptado a um Jar Test, aparelho utilizado em ensaios de floculação, com agitação suficiente para promover maior contato do líquido com a biomassa suspensa. As velocidades de agitação estudadas variaram de 20 a 100 rotações por minuto (rpm), conforme delineamento estatístico. O volume de operação do reator foi de $1 \mathrm{~L}$, considerando-se a soma do volume do efluente nitrificado mais o volume da fonte de carbono, manipueira, que era adicionada no início de cada ciclo operacional, atendendo às relações DQO/N estipuladas no delineamento estatístico, com variação entre 0,1 e 6,3.

A relação $\mathrm{DQO} / \mathrm{N}$ foi calculada de acordo com as características do efluente nitrificado, que

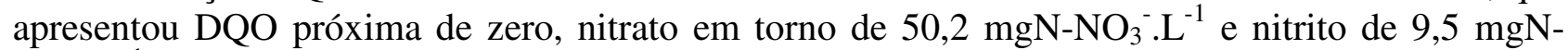
$\mathrm{NO}_{2}{ }^{-} \cdot \mathrm{L}^{-1}$. Dessa forma, na entrada do reator, para cada ciclo operacional, foi considerada apenas a DQO da fonte de carbono (manipueira) com o objetivo de promover a desnitrificação, ou seja, a conversão de aproximadamente $60 \mathrm{mg} \mathrm{L}^{-1}$ de nitrogênio nas formas oxidadas (nitrito e nitrato) a nitrogênio gasoso. Ressalta-se que a manipueira apresentou DQO média de $5.056 \mathrm{mg} \mathrm{L}^{-1}$.

$\mathrm{Na}$ Tabela 1, são apresentadas as relações de DQO/N estudadas, o volume de manipueira utilizado para cada relação, bem como o valor médio da concentração de DQO na mistura (efluente nitrificado mais a manipueira).

TABELA 1. Relação DQO/N, volume de manipueira e concentração de DQO na mistura. COD/N relation, Manipueira volume and $C O D$ concentration in the mix.

\begin{tabular}{ccc}
\hline $\begin{array}{c}\text { Relação } \\
\text { DQO/N }\end{array}$ & $\begin{array}{c}\text { Manipueira } \\
(\mathrm{mL})\end{array}$ & $\begin{array}{c}\text { DQO da mistura } \\
\left(\mathrm{mg} \mathrm{L}^{-1}\right)\end{array}$ \\
\hline 0,1 & 1 & 6,1 \\
1 & 12 & 61,7 \\
3,2 & 38,6 & 196,2 \\
5,4 & 65,2 & 330,7 \\
6,3 & 76,2 & 386,3 \\
\hline
\end{tabular}

O monitoramento analítico envolveu análises físico-químicas de nitrato, nitrito, $\mathrm{pH}$, alcalinidade e temperatura. Os métodos aplicados para determinação destes parâmetros estão 
descritos no Standard Methods for the Examination of Water and Wastewater (APHA, 1998). Os parâmetros nitrito e nitrato foram determinados pelo uso de kits da $\mathrm{HACH}$.

Para o estudo, foi proposto um Delineamento Composto Central Rotacional (DCCR) que permite otimizar o experimento pela redução no número de ensaios, tendo em vista o enorme esforço requerido pelas análises no processo de desnitrificação. Segundo BARROS NETO et al. (2001), essa técnica de planejamento visa a mostrar como os fatores (variáveis envolvidas no processo) podem influenciar uma resposta. A função que descreve tal influência é chamada de superfície de resposta.

Neste estudo, foi avaliada a influência das variáveis independentes e/ou fatores: velocidade de agitação (rpm) e a relação DQO/N sobre o processo de desnitrificação, ou seja, sobre a remoção de nitrato e de nitrito, em porcentagem, uma vez que a agitação rápida pode causar aeração e inibir o processo. Vale ressaltar que o nitrogênio da relação DQO/N, se refere à soma das formas oxidadas, ou seja, $\mathrm{NO}_{2}^{-}$mais $\mathrm{NO}_{3}{ }^{-}$.

Dessa forma, foi realizado um planejamento do tipo Delineamento Composto Central Rotacional (DCCR), com quatro ensaios nos níveis +1 e $-1,4$ ensaios nos níveis dos pontos axiais $(-1,414$ e $+1,414)$ e mais uma triplicata no ponto central $(0)$, totalizando 11 ensaios, realizados em sequência aleatória.

Através de um programa computacional estatístico, foram calculados os principais efeitos dos fatores e suas interações sobre as variáveis respostas que são: remoção de nitrato (\%) e remoção de nitrito (\%), bem como os dados relativos à análise de variância (ANOVA).

\section{RESULTADOS E DISCUSSÃO}

A análise da influência dos fatores objetivou identificar, nas faixas investigadas, as melhores condições para o processo da desnitrificação, através da remoção de nitrato $\left(\mathrm{NO}_{3}{ }^{-}\right)$e nitrito $\left(\mathrm{NO}_{2}{ }^{-}\right)$. $\mathrm{Na}$ Tabela 2, pode ser vista a matriz do planejamento, gerada pela aplicação do Delineamento Composto Central Rotacional (DCCR), com as respectivas variáveis respostas, onde cada linha da matriz representa uma corrida experimental ou ensaio.

TABELA 2. Matriz do planejamento (DCCR) com os fatores (codificados e reais) e resultados obtidos de remoção de nitrato (\%) e remoção de nitrito (\%).Matrix Planning (CCRD) with the factors (coded and real) and results of nitrate $(\%)$ and nitrite (\%) removal.

\begin{tabular}{ccccccc}
\hline \multirow{2}{*}{$\begin{array}{c}\text { Números de } \\
\text { Ensaios }\end{array}$} & \multicolumn{2}{c}{$\begin{array}{c}\text { Fatores } \\
\text { (Valores Codificados) }\end{array}$} & \multicolumn{2}{c}{$\begin{array}{c}\text { Fatores } \\
\text { (Valores Reais) }\end{array}$} & \multicolumn{2}{c}{ Variáveis Respostas } \\
\cline { 2 - 7 } & $\begin{array}{c}\text { Relação } \\
\text { DQO/N }\end{array}$ & Agitação & $\begin{array}{c}\text { Relação } \\
\text { DQO/N }\end{array}$ & $\begin{array}{c}\text { Agitação } \\
(\text { rpm) }\end{array}$ & $\begin{array}{c}\text { Remoção } \\
\text { de } \\
\text { Nitrato (\%) }\end{array}$ & $\begin{array}{c}\text { Remoção } \\
\text { de } \\
\text { Nitrito (\%) }\end{array}$ \\
\hline 1 & -1 & -1 & 1 & 32 & 75,1 & 75,0 \\
2 & 1 & -1 & 5,4 & 32 & 100,0 & 66,7 \\
3 & -1 & 1 & 1 & 88 & 63,4 & 66,7 \\
4 & 1 & 1 & 5,4 & 88 & 93,5 & 100,0 \\
5 & $-1,414$ & 0 & 0,1 & 60 & 14,3 & 0,0 \\
6 & 1,414 & 0 & 6,3 & 60 & 85,6 & 66,7 \\
7 & 0 & $-1,414$ & 3,2 & 20 & 100,0 & 75,0 \\
8 & 0 & 1,414 & 3,2 & 100 & 93,5 & 100,0 \\
9 & 0 & 0 & 3,2 & 60 & 100,0 & 100,0 \\
10 & 0 & 0 & 3,2 & 60 & 100,0 & 100,0 \\
11 & 0 & 0 & 3,2 & 60 & 100,0 & 100,0 \\
\hline
\end{tabular}

A partir dos dados acima, foram gerados os gráficos de Pareto (Figura 1), no qual apenas uma variável independente estudada mostrou influência na remoção de nitrato (\%) e remoção de nitrito 
(\%). O diagrama de Pareto ilustra graficamente a influência das variáveis independentes sobre as variáveis respostas, considerando-se significativas aquelas cujas colunas horizontais ultrapassam as linhas tracejadas, representativas para intervalo de confiança de $95 \%(\mathrm{p}<0,05)$.

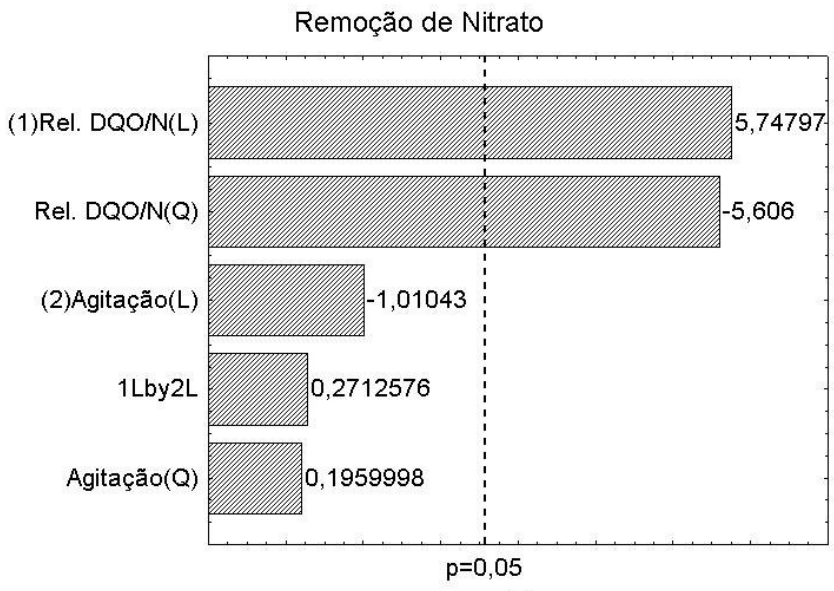

(a)

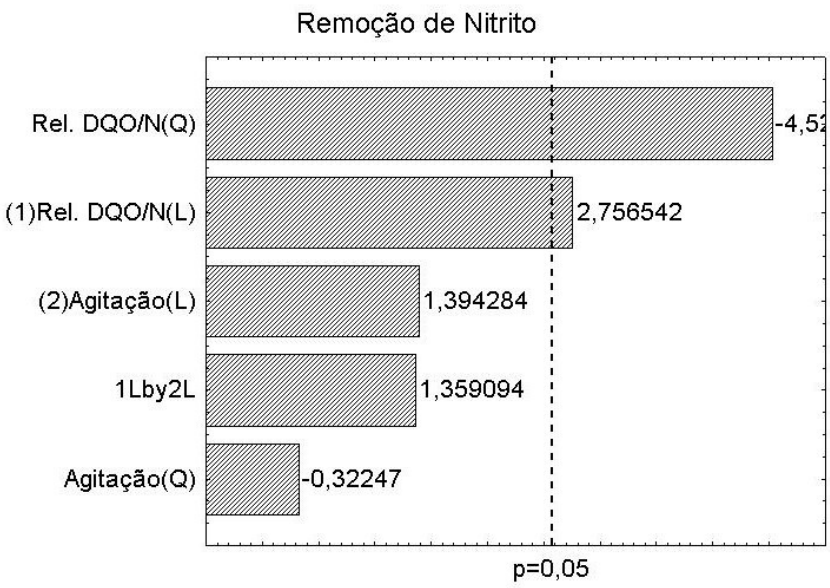

(b)

FIGURA 1. Gráfico de Pareto para remoção de nitrato (a) e remoção de nitrito (b). Pareto chart for the nitrate (a) and nitrite (b) removal.

Portanto, pode-se afirmar que a relação $\mathrm{DQO} / \mathrm{N}$ apresentou efeito significativo sobre a remoção de nitrato e a remoção de nitrito, enquanto a velocidade de agitação e a interação dos dois fatores não tiveram efeito significativo, para um nível de 5\% de significância.

Dessa forma, gerou-se um modelo quadrático (equação matemática) para as variáveis respostas, representados pelas equações 1 e 2, com coeficientes de regressão de $93,27 \%$ e $86,84 \%$, respectivamente. Esses valores mostram que mais de $90 \%$ da variação na remoção de nitrato (\%) é explicado pelo modelo apresentado, enquanto para nitrito esse valor não ultrapassa $87 \%$.

Remoção de Nitrato $(\%)=99,998+19,480\left(\right.$ Rel. DQO/N) -22,616 (Rel. DQO/N) ${ }^{2}$

Remoção de Nitrito $(\%)=99,997+14,917\left(\right.$ Rel. DQO/N) - 29,160 (Rel. DQO/N) ${ }^{2}$

Ressalta-se que, nessas equações, é considerado o fator relação $\mathrm{DQO} / \mathrm{N}$ na sua forma codificada. Através da análise de variância (ANOVA), apresentada na Tabela 3, observou-se que os modelos mostraram boa concordância com os dados experimentais obtidos.

TABELA 3. ANOVA dos modelos quadráticos da remoção de nitrato (\%) e da remoção de nitrito (\%). ANOVA of the quadratic models of nitrate (\%) and nitrite (\%) removal.

\begin{tabular}{|c|c|c|c|c|c|c|c|}
\hline & $\begin{array}{l}\text { Fonte de } \\
\text { Variação }\end{array}$ & $\begin{array}{l}\text { Soma dos } \\
\text { Quadrados }\end{array}$ & $\begin{array}{l}\text { Graus de } \\
\text { Liberdade }\end{array}$ & $\begin{array}{l}\text { Quadrado } \\
\text { Médio }\end{array}$ & $\mathrm{F}_{\text {calculado }}$ & $\mathrm{F}_{\text {tabelado }}$ & $\mathrm{R}^{2}$ \\
\hline \multirow{2}{*}{ 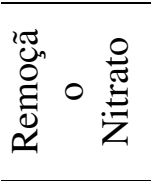 } & $\begin{array}{c}\text { Regressão } \\
\text { (modelo) }\end{array}$ & 6365,182 & 5 & 1273,036 & 13,86 & 5,05 & 0,9327 \\
\hline & Resíduos & $\begin{array}{r}459,360 \\
682450\end{array}$ & 5 & 91,872 & - & - & - \\
\hline \multirow{2}{*}{ 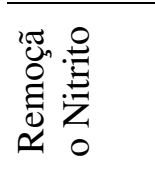 } & $\begin{array}{c}\text { Regressão } \\
\text { (modelo) }\end{array}$ & 7728,286 & 5 & 1545,657 & 6,599 & 5,05 & 0,8684 \\
\hline & $\begin{array}{c}\text { Resíduos } \\
\text { Total }\end{array}$ & $\begin{array}{l}1171,110 \\
8899,396\end{array}$ & $\begin{array}{c}5 \\
10\end{array}$ & $\begin{array}{c}234,22 \\
-\end{array}$ & - & - & $\begin{array}{l}- \\
-\end{array}$ \\
\hline
\end{tabular}

Verificou-se que os modelos quadráticos são válidos no intervalo de $95 \%$ de confiança, tendo em vista que o $\mathrm{F}_{\text {calculado }}$ é maior que o $\mathrm{F}_{\text {tabelado, }}$ o que torna esses modelos passíveis de serem usados para estimar valores para a resposta quanto à remoção de nitrato (\%) e remoção de nitrito, a partir das superfícies de resposta. 
$\mathrm{Na}$ Figura 2, encontram-se as superfícies de resposta obtidas para o processo da desnitrificação.

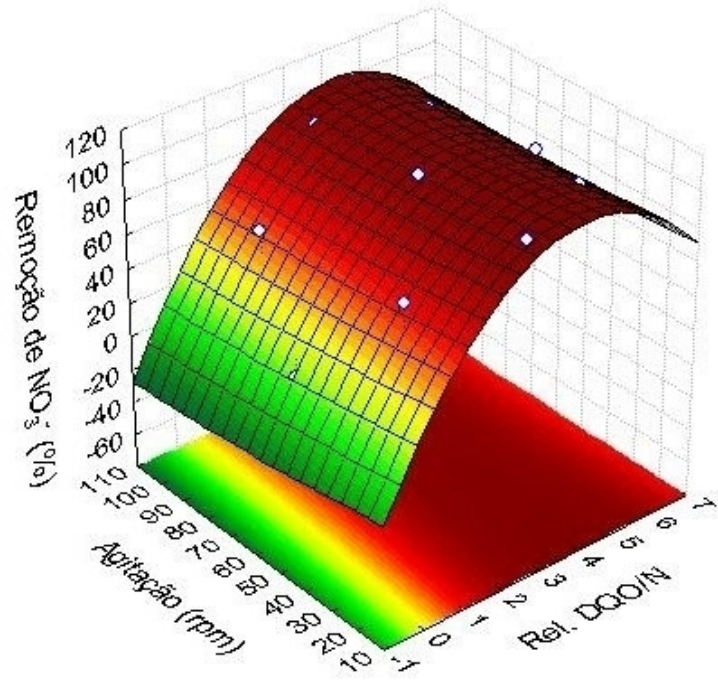

(a)

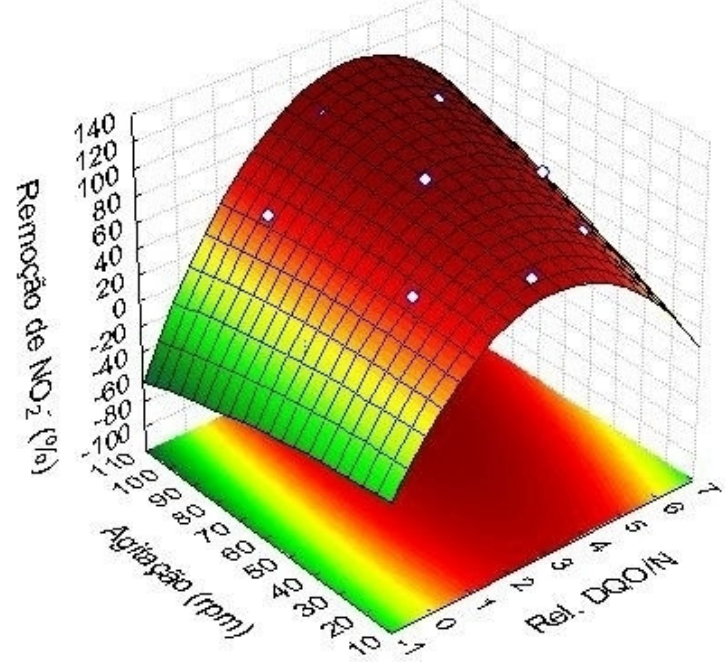

(b)

FIGURA 2. Superfície de resposta para a remoção de nitrato (a) e remoção de nitrito (b), em função da velocidade de agitação e relação DQO/N. Response surface for the nitrate (a) and nitrite (b) removal as a function of agitation speed and COD/N.

A partir da avaliação das eficiências de remoção de nitrato e de nitrito (Figura 2(a) e (b), respectivamente), constatou-se que a agitação não apresentou efeito significativo sobre o processo de desnitrificação (remoção das formas oxidadas: nitrito e nitrato). Entretanto, SÀNCHEZ et al. (2000) encontraram efeito da agitação sobre o processo de desnitrificação. Os autores estudaram os efeitos da relação $\mathrm{C} / \mathrm{N}$ (1,3 e 2) e agitação (0 e $180 \mathrm{rpm})$, na determinação da atividade desnitrificante de um esgoto sintético. Tais parâmetros foram avaliados com o objetivo de se estabelecerem as condições operacionais ótimas. A máxima atividade desnitrificante obtida foi com relação $\mathrm{C} / \mathrm{N}$ de 1,3 e frascos agitados a $180 \mathrm{rpm}$, sendo observada, neste caso, completa desnitrificação a nitrogênio molecular (98-99\% de nitrogênio molecular na composição do gás), em um período de 12 horas.

A relação DQO/N teve efeito significativo, ao nível de 5\% de significância, com eficiência de remoção de $100 \%$, nas faixas compreendidas entre 3,2 e 5,4, relação esta maior que a encontrada por SÀNCHEZ et al. (2000).

Observou-se que, com o aumento da relação DQO/N, houve um aumento da remoção até um ponto ótimo ( $100 \%$ de remoção) e, à medida que a relação $\mathrm{DQO} / \mathrm{N}$ aumentou, ocorreu consequente queda, tanto na remoção de nitrato (\%) quanto na remoção de nitrito (\%). Neste caso, o aumento da relação $\mathrm{DQO} / \mathrm{N}$ pode ter favorecido o crescimento de outros microrganismos, gerando competição com os microrganismos desnitrificantes.

Estudo semelhante foi realizado por HER \& HUANG (1995), que avaliaram algumas fontes de carbono (ácido acético, glicose, ácido benzoico e metanol) em diferentes relações $\mathrm{C} / \mathrm{N}$, no processo de desnitrificação de esgoto sintético nitrificado, através da remoção das formas oxidadas de nitrogênio (nitrito e nitrato). Para completa desnitrificação (98 a 100\%), os autores supracitados encontraram uma relação máxima e mínima requerida para cada fonte de carbono e observaram crescimento linear da desnitrificação, que atingiu um patamar no qual a remoção de nitrogênio se manteve constante (aproximadamente 98,8\%), até atingir um valor de relação C/N no qual houve queda na taxa de desnitrificação. Os autores relacionaram essa queda ao peso molecular dos compostos utilizados como fonte de carbono. 
Durante os ensaios realizados, foram também monitorados parâmetros como temperatura, $\mathrm{pH}$ e alcalinidade $\left(\mathrm{CaCO}_{3}\right)$, tendo em vista que os mesmos podem exercer influência sobre o processo de desnitrificação.

Segundo VAN HAANDEL \& MARAIS (1999), a desnitrificação aumenta com a temperatura até o valor próximo a $40{ }^{\circ} \mathrm{C}$. A temperatura média do ambiente de trabalho foi de $27{ }^{\circ} \mathrm{C}$, o que favoreceu o crescimento dos microrganismos desnitrificantes, pois em todos os ensaios ocorreu a remoção de nitrato e nitrito, em maior ou menor quantidade. A temperatura está na faixa reportada por SURAMPALLI et al. (1997), que citaram faixas ótimas de trabalho entre 10 e $30^{\circ} \mathrm{C}$.

Os parâmetros $\mathrm{pH}$ e alcalinidade apresentaram o comportamento esperado, tendo em vista que a produção de alcalinidade durante a conversão de nitrato e nitrito a nitrogênio gasoso implica o aumento do $\mathrm{pH}$. Diversos autores, dentre os quais SOUSA \& FORESTI (1999), citam faixa ótima de $\mathrm{pH}$ entre 6,5 e 8,0. Em média, o pH no início de ciclo foi de 7,6, e no final, de 8,2, com exceção do ensaio 5, cujo $\mathrm{pH}$ na entrada se encontrava com valor de 8,5. VAN HAANDEL \& MARAIS (1999) relatam que, para pH menor que 6,0 e maior que 8,5, há uma grande redução da atividade de desnitrificação. Entretanto, o efeito negativo no processo de desnitrificação para o ensaio 5 deve-se à baixa relação $\mathrm{DQO} / \mathrm{N}$ utilizado nesse ensaio. Os valores de alcalinidade variaram, em média, $321 \mathrm{mg} \mathrm{L}^{-1}$ no início e $391 \mathrm{mg} \mathrm{L}^{-1}$ no final de ciclo, o que evidenciou a produção de alcalinidade no processo.

\section{CONCLUSÕES}

A manipueira, quando adicionada na fase anóxica, apresentou-se como uma fonte viável para o processo de desnitrificação de efluente de abatedouro de tilápia, tendo em vista que, para todos os ensaios, ocorreu remoção de nitrato e de nitrito em maior ou menor quantidade, num período de 4 horas.

A relação $\mathrm{DQO} / \mathrm{N}$ teve influência significativa sobre o processo de desnitrificação, com faixa ótima de operação entre 3,2 e 5,4, em que foram obtidas eficiências de remoção de nitrogênio de $100 \%$.

Constatou-se que a agitação não exerceu influência significativa, no intervalo de confiança de $95 \%$, sobre o processo da desnitrificação.

A temperatura média do ambiente de trabalho não teve efeito inibitório sobre a atividade dos microrganismos desnitrificantes, e os parâmetros $\mathrm{pH}$ e alcalinidade $\left(\mathrm{CaCO}_{3}\right)$, monitorados durante todos os experimentos, apresentaram comportamento esperado para o processo da desnitrificação, evidenciando a produção de alcalinidade.

\section{AGRADECIMENTOS} financeiro.

À Fundação Araucária da Secretaria de Ciência e Tecnologia do Estado do Paraná, pelo apoio

\section{REFERÊNCIAS}

AMERICAN PUBLIC HEALTH ASSOCIATION - APHA; AMERICAN WATER WORKS ASSOCIATION - AWWA; WATER ENVIRONMENT FEDERATION - WPCF. Standard methods for the examination of water and wastewater. $20^{\text {th }} \mathrm{ed}$. Washington, 1998.

BARROS NETO, B.; SCARMINIO, I.S.; BRUNS, R.E. Como fazer experimentos: pesquisa e desenvolvimento na ciência e na indústria. Campinas: Editora da UNICAMP, 2001.

FERREIRA, E. Cinética química e fundamentos dos processos de nitrificação e denitrificação biológica. In: CONGRESSO INTERAMERICANO DE ENGENHARIA SANITÁRIA E AMBIENTAL, 27., 2000, Porto Alegre. Anais... Rio de Janeiro: ABES, 2000. 1 CD-ROM. 
HER, J.; HUANG, J. Influences of carbon source and C/N ratio on nitrate/nitrite denitrification and carbon breakthrough. Bioresource Technology, Taiwan, v.54, p.45-51, 1995.

ISOLDI, L.A.; KOETZ, P.R. Tratamentos biológicos para remoção de matéria carbonada e nitrogenada. Revista Eletrônica do Mestrado em Educação Ambiental, Rio Grande, v.12, p.1-12, 2004.

KIRSCHNIK, P.G. Avaliação da estabilidade de produtos obtidos de carne mecanicamente separada de tilápia nilótica (Oreochromis niloticus). 2007. 91 f. Tese (Doutorado em Aquicultura) - Universidade Estadual Paulista, Centro de Aquicultura, Jaboticabal, 2007.

KUBIZTA, F. Tilápia: tecnologia e planejamento na produção comercial. Jundiaí: Fernando Kubitza, 2000. 289 p.

SÁNCHEZ, M.; MOSQUERA-CORRAL, A.; MÉNDEZ, R.; LEMA, J.M. Simple methods for the determination of the denitrifying activity of sledges. Bioresource Technology, Essex, v.75, p.1-6, 2000.

SOUSA, J.T.; FORESTI, E. Utilização de lodo anaeróbio como fonte externa de carbono no processo de desnitrificação de águas residuárias. Revista Brasileira de Engenharia Agrícola e Ambiental, Campina Grande, v.3, n.1, p.69-73, 1999.

SURAMPALLI, R.Y.; TYAGI, R.D.; SCHEIBLE, O.K.; HEIDMAN, J.A. Nitrification, denitrification and phosphorus removal in sequential batch reactors. Bioresource Technology, Essex, v.61, p.151-157, 1997.

VAN HAANDEL, A.; MARAIS, G. O comportamento do sistema de lodo ativado: teoria e aplicações para projeto e operação. Campina Grande: Universidade Federal da Paraíba, 1999. 